\title{
Development of an Automatic Master-batch Dispensing System with Iterative Learning Control
}

\author{
Peigang Li, Xianying Feng ${ }^{*}$, Fuxin Du, Wenping Liu and Jian Zheng \\ Shandong University, Jinan, Shandong Province, China, 250061 \\ ${ }^{*}$ Corresponding author
}

\begin{abstract}
Based on IPC and PLC, a distributed master-slave precision dispensing control system in master-batch production is developed. IPC is used to manage the information related to material, formula, process, and production, and to monitor the whole process of proportioning. PLC is used to control specific motions. A new iterative learning method, together with the overall control scheme, the software and hardware architecture are proposed. Practical operation proves that the system is feasible, reliable, interface-friendly, simple in operation and accurate in proportioning.
\end{abstract}

Keywords-automatic dispensing; iterative learning control; PLC; drop value

\section{INTRODUCTION}

Automatic dispensing is an accurate metering pr-ocess of raw materials based on the preset proportion. It is very important for the production of chemical, pharmaceutical, building materials, food and other industries. The dispensing efficiency imposes a heavy impact on the productivity of the entire production line, and the proportioning precision of ingredients directly affects the quality and property of products [1-3]. However, traditional PID control of the controller parameter adjustment is inconvenient and cannot be self-tuned. Huijuan Yi adopted fuzzy control in the weighing system [4]. Due to the difficulty in obtaining the control law, the system cannot be adjusted online once it has been preset.

In the paper, a master-slave distributed control system consisting of a management PC, and manipulation control PLC is built. To ensure the dispensing accuracy, stage speed are used during the feeding process. Iterative learning method with forgetting factor is used for dynamic correction of measurement errors.

\section{PRODUCTION FLOW}

The system is equipped with a powder scale and a liquid scale, capable of metering 3 types of powder materials and 4 types of liquid materials. Due to different materials share a single scale, the ingredients are measured and calculated time and again. The material warehouse opens and the corresponding screw conveyor is activated according to the specific ingredient in the formulation. When the weighing value reaches a certain value, the screw conveyor stops and the material bin valve is closed. Similar cycle will be followed for the measurement of the rest raw materials. Finally, the high-speed mixing machine is expected to receive material, according to the specified order in the formula to discharge and then the proportioning is finished. Ingredients are loaded into the high-speed machine for full mixing, and finally pass through the internal mixer before discharge. The production process is shown in Figure. 1.

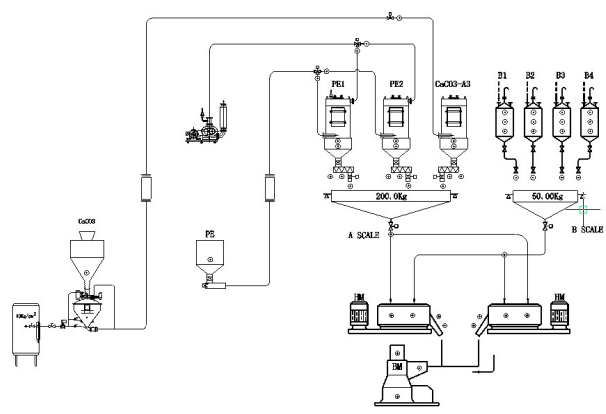

FIGURE I. THE PRODUCTION PROCESS

There is a distance between the material warehouse valves and the scale. Once the material warehouse valves open, the materials fall into the scale with a certain delay. After the silo valve closes, there will be a portion of the material that do not fall into the scale. The weight between the silo and the scale "freedom" stock column is called drop value. For accurate weighing, it is necessary to properly close the valve in advance. When the scale indicates the set value minus the drop value, the silo valve will close and the set value is reached after the air material falls completely. It can be seen that the drop value directly affects the accuracy of the system. In order to achieve accurate dispensing, constant correction to the drop value will be required.

\section{SYSTEM ARCHITECTURE AND CONTROL ALGORITHM}

\section{A. System Architecture}

According to the requirements for master-batch production process and dispensing accuracy, the "IPC+PLC" control pattern is devised as shown in Fig. 2. It consists of 3-level network control system of IPC-610MB by Yan hua Co., S7-300 PLC and SIWAREX-U weighing module by Siemens, PT1041B Touch screen by Panel Master, and CIMRHB4A0006A yaskawa inverter. PLC connects inverter through PROFIBUS$\mathrm{DP}$ and connects weighing module through RS232. The communication between IPC and PLC is completed through the PRODAVE tool provided by SIEMENS to complete communication with PLC. IPC is in charge of online monitoring of the whole dispensing process. With $\mathrm{CH}$ advanced programming in Windows XP environment and SQL database, historical data query can be conducted in the background [5].

IPC completes the following aspects of the function, mainly including the whole process monitoring, alarm detection, 
production report statistics, video monitoring, real-time parameter display and so on. PLC mainly completes the function of the action execution, the equipment state information feedback and so on.

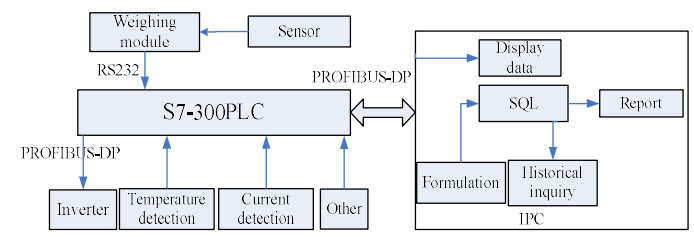

FIGURE II. SCHEMATIC DIAGRAM OF CONTROL SYSTEM STRUCTURE

\section{B. Feeding Control Algorithm}

The relationship between the actual value of the weighing scales $W_{\mathrm{S}}$ and set the value of $W_{\mathrm{d}}$ and drop value $\mathrm{U}$ is written in the following equation [6].

$$
W_{s}=(\rho S k-1) U+(1-\rho S k) W_{\mathrm{d}}+\rho S H
$$

where

$\rho$ - the proportion of materials in air $(\mathrm{kg} / \mathrm{m} 3)$

$S$ - the cross section area of the remaining material $\left(\mathrm{m}^{2}\right)$

$K$ - ratio coefficient $(\mathrm{m} / \mathrm{kg})$

$H$ - the distance between the valve of the material and the scale at the bottom

$S, H$ and $k$ are constants, only $\rho$ is difficult to determine, by the external environmental factors, but in a period of time can be seen as relatively constant.

Iterative learning is an attempt to control the system to be controlled, through the control to the output signal and the deviation of the set target to correct the imperfect control signal, to gradually improve the tracking performance of the system. In a given time slot $[0, \mathrm{~T}]$, the desired trajectory $y_{d}(\mathrm{t})$ The error $\left(e^{e_{k}(\mathrm{t})}\right)$ is generated in the $\mathrm{k}-\mathrm{th}$.

$$
e_{k}(\mathrm{t})=y_{d}(\mathrm{t})-y_{k}(\mathrm{t})
$$

Then the recursive formula of the general form of the iterative learning control law can be expressed as

$$
u_{k+1}(\mathrm{t})=L\left(u_{k}(\mathrm{t}), e_{k}(\mathrm{t})\right)
$$

The basic schematic diagram of the iterative learning control is shown in Figure. 3.

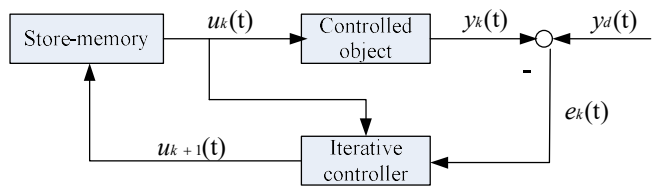

FIGURE III. INTERATIVE LEARNING CONTROL SCHEMATIC

There are a number of affecting factors in practical production, such as the air humidity, and the uniformity of materials. Some affecting factors are tentative. So this system must be stable. To improve the speed of the iterative learning control and the stability of the iterative control, this paper uses a new iterative control strategy with forgetting factor proposed by Xie Sheng li et al. Learning control algorithm is as follows[7]:

$$
u_{k+1}(\mathrm{t})=(1-\alpha) u_{k}(\mathrm{t})+\alpha u_{k-1}(\mathrm{t})+L e_{k}(\mathrm{t}), \quad k=0,1,2, \cdots
$$

From Equation (1), the system control is invariant. Although there are uncertain model emerged from uncertain parameters $\rho$, it can be controlled through the training of learning. By the repeated training of the drop value $U$, it makes the system's actual weighing value Ws gradually approaching the set value Wd. According to the idea of iterative control.

Each measurement desired trajectory $y_{d}(\mathrm{t})$ is given in the interval $[\mathrm{t} 1, \mathrm{t} 2]$, and then through the output error $e(\mathrm{t})$ to calculate the next measurement of the drop value, so that the actual value of the weighing approximation set value.

Ingredients for the first time, when the scale of the measurement value reached $\mathrm{W}_{\mathrm{d}}-\mathrm{U}_{0}$, material valve is closed, the final weighing the actual value is $\mathrm{W}_{0}$, then The error between actual value and set value is shown as the following formula.

$$
e_{0}=W_{0}-W_{d}
$$

The error produced in the measurement of the $\mathrm{k}$ can be expressed as

$$
e_{k}=W_{k}-W_{d}
$$

From formula (2), New drop value can be produced.

$$
U_{1}=(1-\alpha) U_{0}+\alpha \mathrm{U}_{-1}+L e_{0}
$$

$\alpha$ Forgetting-factor $0 \leq \alpha<1$, L Weighted learning factor $0<L<1$, make $\mathrm{U}_{0}$ equal to $\mathrm{U}_{-1}$ in the first iteration. Then according to the forgotten iterative control algorithm, the error predicted in the measurement of the $k+1$ can be expressed as

$$
U_{k+1}=(1-\alpha) U_{k}+\alpha U_{k-1}+L e_{k}
$$

From Equation (3) we know that in order to improve the convergence rate when the error is large, larger values $L$ should be selected; when the error is small, smaller values $L$ should be selected. Therefore, an inverse correlation function is constructed:

$$
L_{k}=\frac{\left|e_{k}\right|}{\left|e_{k}\right|+M} \quad(\mathrm{M} \text { as constant })
$$

$M$ reflects the sensitivity of the system to the error. With the increase of $\mathrm{M}$, the sensitivity of the system is lower. $M$ should be selected according to the actual situation. In addition, to ensure $0<U_{k}<W_{d}$, boundary detection is performed in the iterative process.

For $a=0.1, M=0.1, W_{\mathrm{d}}=100 \mathrm{~kg}, S=0.1 \mathrm{~m}^{2}, U_{0}=2 \mathrm{~kg}, H=2 \mathrm{~m}$, 
$k=0.001 \mathrm{~m} / \mathrm{kg}, \rho=20( \pm 0.05) \mathrm{kg} / \mathrm{m}^{3}$, the simulation results of MATLAB combined with $\mathrm{C} \#$ are shown in Figure. 4.

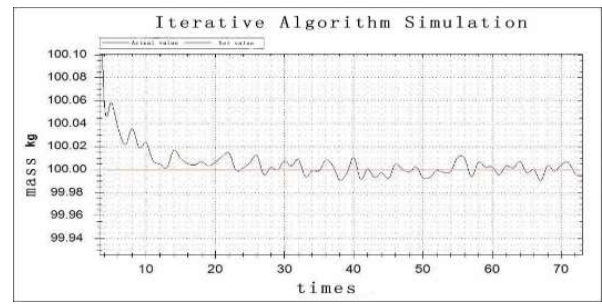

FIGURE IV. SIMULATION RESULT

After 9 generations of iteration, the actual $v$ alue of the $10^{\text {th }}$ weighing has reached $100.018 \mathrm{~kg}$, after basically stable in $100 \pm$ $0.02 \mathrm{~kg}$, the pass rate of $98.8 \%$, which meets the quality requirements of production enterprises.

\section{MAIN SOFTWARE DESIGN AND IMPLEMENTATION OF CONTROL SYSTEM}

\section{A. The Design of PC Program}

The host computer acts as the medium between the user and the slave computer. The traditional configuration is difficult to meet the flexible production requirement. The system combined with the actual problem of the burden of production, with VS2010 as the development tool in the host computer, achieve the warehouse material, the content of the management, as well as online monitoring of the whole process of batching. The main function is shown in Figure. 5. User management is used to check the identity of the operator as well as the authority of the management. Process management is a set of production process parameters, including the production formula, schedule planning, etc. Process monitoring mainly includes the collection of the actual data in the production process, the status of the equipment display, alarm detection and video monitoring of each station. Data report is statistical information on the production of products, raw materials, manpower and so on. Database management mainly includes the backup, restore and cleanup of the database. Digital camera video surveillance using Hikvision achieves the key position monitor. The main interface of the software operation is shown in Figure. 6.

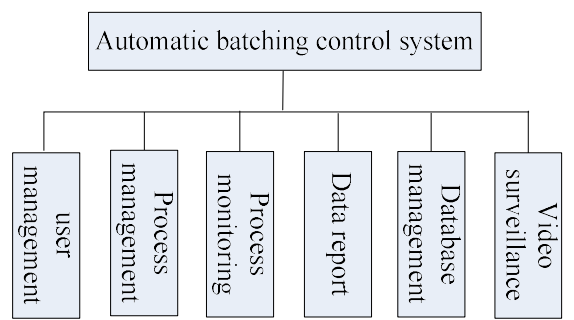

FIGURE V. SYSTEM FUNCTION DIAGRAM

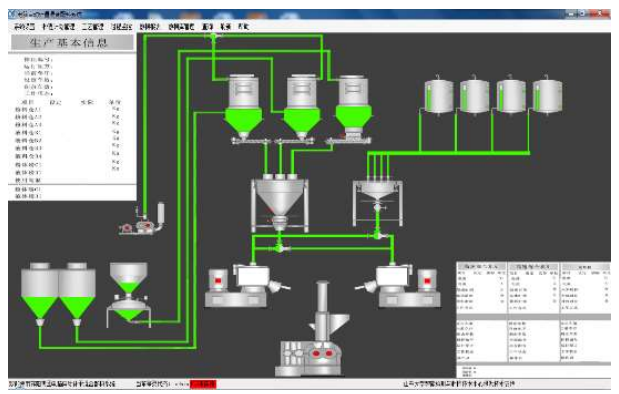

FIGURE VI. OPERATING MAIN INTERFACE

\section{B. Communication between IPC and PLC}

This control system solves the problem of IPC and PLC communication through the MPI/IE PRODAVE 6.2 module provided by SIEMENS. MPI/IE PRODAVE 6.2 is designed to solve the problem of SIEMENS PC and SIEMENS PLC communication tools. It can be carried out with the SIEMENS S7 series PLC data transmission, by the "DB_Read/Write", "Read/Write Field" method. PRODAVE provides a rich Windows operating system based on the DLL function, for the PLC and PC communications provides a great convenience.

These functions can be called in the VS development environment to establish a communication connection with PLC.PROD-AVE realization of PC and PLC communication not only read and write with high speed, high transmission accuracy, but also do not need to write communication program in PLC. The communication process is shown in Figure. 7.

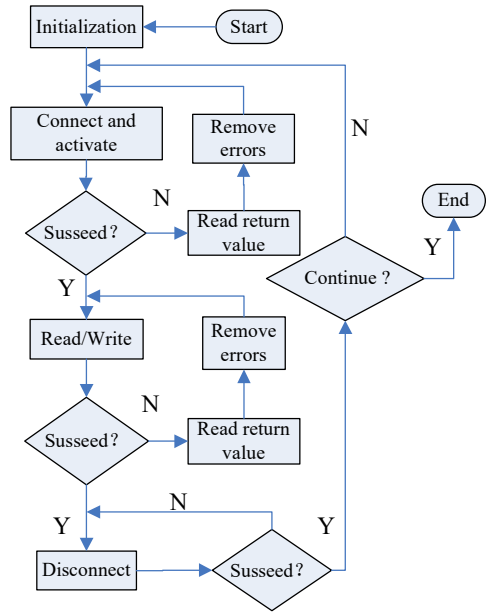

FIGURE VII. COMMUNICATION FLOW CHART

\section{CONCIUSION}

With the stage-speed feeding and forgetting factor of the iterative learning algorithm for dispensing control, the system realizes the close-loop real-time dynamic error adjustment, improves the measurement accuracy, and guarantees the measurement speed. The system has been successfully applied to the practical production. It is proved that the system is capable of fast and accurate dispensing control, with a control accuracy less than $\pm 0.02 \%$, qualified rate higher than $98 \%$. Practical operation proves that the system is feasible, reliable, interface-friendly, simple in operation and accurate in proportioning. 


\section{REFERENCES}

[1] M Hong, Y Yu-Xiang, W Zhong. Automatic batching system for cement based on PLC[J]. PP.128-130. 2013(01):

[2] P Hai-Liang, P Feng. Design of Blending System for Liquid Washing Machine[J]. Instrument Technique and Sensor. PP.81-85.2015(07).

[3] Hang B, Jiang L, Sun L. Parameter Self-Tu ning Based Industrial Batching and Weighting Control System[J]. International Journal of Innovative Computing Information and Control. 2007.

[4] Y Huijuan, Z Xiaojiang, Y Maojin, et al. Design and research on fuzzy control in weighing system of polyurethane resin batching[J]. Modern Manufacturing Engineering. PP4-7. 2015(10).

[5] W Jia Yin, F Xian Ying, W Xiao Bin. Development of Precise Quantitative Packaging Control System for Reagent Based on PLC[J]. Modular Machine Tool \& Automatic Manufacturing Technique. PP. 8082. 2013(10).

[6] W U Ming-Liang, G Peng-Peng, G Yun-Ying, et al. Research of Automatic Batching Control System Based on PLC [J]. Automation \&amp; Instrumentation. PP. 44-47. 2013, 28(4).

[7] T Sen ping, X Sheng li. A New Iterative Learning Control Algorithm with Forgetting Factor[C]. Wuhan Industrial University Press, 2003 